\title{
Three-Dimensional Application-Specific Protocol Architecture for Wireless Sensor Networks
}

\author{
Mostafa Baghouri ${ }^{1}$, Abderrahmane Hajraoui ${ }^{2}$, Saad Chakkor ${ }^{3}$ \\ Department of Physics, Communication and detection systems laboratory, \\ Faculty of Sciences, University of Abdelmalek Essaâdi, Tetouan, Morocco \\ *Corresponding author, e-mail: baghouri.mostafa@gmail.com', ad_hajraoui@hotmail.com², \\ saadchakkor@gmail.com ${ }^{3}$
}

\begin{abstract}
Many researchers assume that the distribution of the nodes is done in a two-dimensional environment in the reason that the height of this network is negligible compared to its width and length. However, in the reality, three-dimensional (3D) deployment of the wireless sensor networks is used. Therefore, many applications require 3D architecture such as underwater, space communications, atmospheric, forest or building. Unfortunately, the energy consumption and throughput in the $3 D$ environment decreases considerably compared to 2D in which we can't neglect them in some applications. In his paper we applied the 3D architecture in $L E A C H$ protocol and we prove by computer simulation how this $2 D$ approximation is not reasonable since the lifetime of $3 D$ WSN decrease by about $21 \%$ over than 2D WSN.
\end{abstract}

Keywords: wireless sensor networks, LEACH protocol, Energy-efficiency, 2D and $3 D$ WSN, network lifetime

Copyright $\odot 2015$ Institute of Advanced Engineering and Science. All rights reserved.

\section{Introduction}

In the reality the physical world we live in, is a 3D envi-ronment. Therefore, many applications, such as underwater, underground, airborne, space communications, atmospheric, forest, body or building, of WSN deployed in three-dimensional space (see Figure1). A wireless sensor network (WSN) is considered as three-dimensional (3D) when the height of deployed sensor nodes field is not negligible as com-pared to length and breadth of network [1]. However, with the complexity of the design and analysis of the 3D WSN, wireless sensor network in 2D plane are more studied than in 3D space.

A 3D wireless sensor network is a set wireless sensor nodes distributed in a 3D plane. Each sensor node has emission to sense the events detection, such as temperature, pressure or vibration and send their measurements toward a processing center called sink [1, 2]. Due to the limitation in their battery capacity which their replacement is impossible, optimization of this unique resource has become an important issue. Node clustering is an effective technique for improving the energy efficiency and prolonging the network lifetime of a WSN [3] and has been widely studied in 2D WSNs.

$\operatorname{LEACH}[3,4]$ is one of the first protocols which use this technique and has been applied into the underwater environ-ment by doing some changes [5-8]. All of these literatures considered that the nodes are distributed in tow-dimensional area.

In this paper, we show that approximate the 3D field in the 2D environment deployment is not negligible when a height of network is greater.

The rest of the paper organization is done as follows: Section II summarizes the related work. Three-dimensional wire-less sensor network model is provided in section III. The Simulation results are carried out in section IV. Finally we conclude our research work and give some perspectives in section $\mathrm{V}$. 


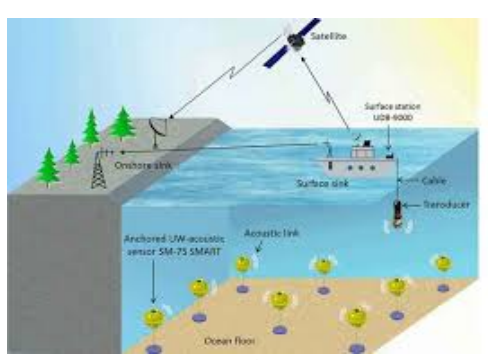

a)

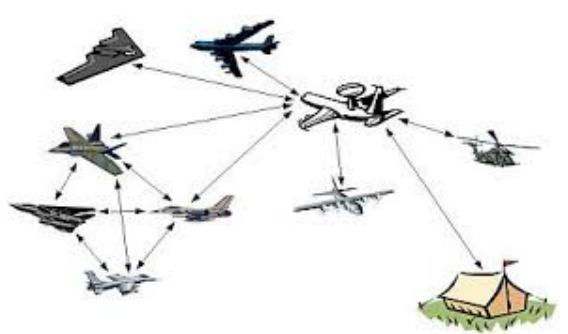

c)

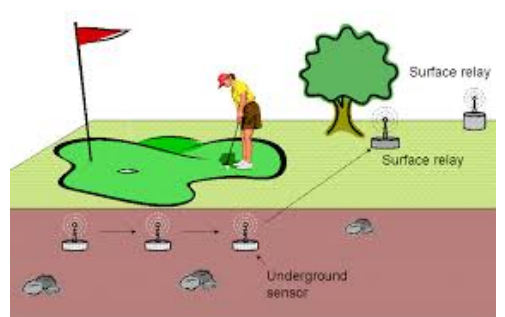

b)

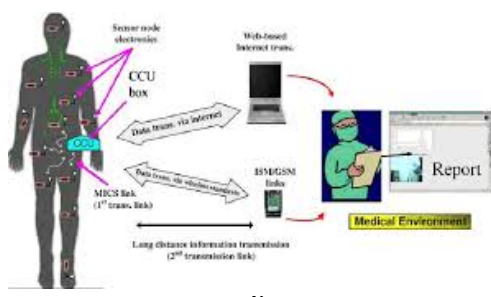

d)

Figure 1. Examples of three-dimensional wireless sensor networks: a) underwater, b) underground, c) airborne, and d) body

\section{Related Work}

Some works try to use the existing WSN clustering protocol for WSN in underwater environment. Reference [6] assumed UASNs are less dynamic than normal WSNs and proposed the LEACH-L, which updates its state locally, and reduced the overhead of LEACH. In [9], a clustering scheme is proposed in the context of routing scheme to extend the lifetime of UASN. Reference [10] designed a cluster structure without considering energy problem. Gu et al [11] have presented a feasible routing protocol for underground WSN in coal mine, called LEACH-mine. In the algorithm, all nodes are located in three sides of the XY projection plane and in the internal of the rectangular of the $X Z$ projection plane. Zhou et al $[12,13]$ have deployed a 2D WSN for coal mine, comparing to the random node deployment strategy, the strategy proposed in this work can prolong the life by two times. However, they have not considered the influence of height of the network.

Generally, in the practical applications of WSN, the sensor nodes need to be deployed and communicate in the three-dimensional area in the order to monitoring the hostile regions such as underwater, underground mine, airborne, and body environments. Therefore, to more approach to the reality situations, a 3D WSN deployment is studied dentally in this paper.

Based on the analysis above, we find that few works on 3D deployment have been studied for WSNs. Driven by this observation; we will show by simulation that these assumptions and approximations are not reasonable in some applications of WSN.

\section{Three Dimensional Wireless Sensor Network Models}

\subsection{Energy Model}

This study assumes a simple model for the radio hardware where the transmitter dissipates energy for running the radio electronics to transmit and amplify the signals, and the receiver runs the radio electronics for reception of signals [7]. Multipath fading model $\left(\mathrm{d}^{4}\right.$ power loss) for large distance transmissions and the free space model ( $\mathrm{d}^{2}$ power loss) for proximal transmissions are considered. Thus to transmit an $l-$ bits message over a distance $\mathrm{d}$, the radio expends:

$$
\begin{aligned}
& E_{T x}(l, d)=E_{T x-e l e c}(l)+E_{T x-a m p}(l, d) \\
& E_{T x-e l e c}(l)=l E_{\text {elec }}
\end{aligned}
$$




$$
E_{T x-a m p}(l, d)=\left\{\begin{array}{l}
l \epsilon_{f s} d^{2}, \text { when } d<d_{0} \\
l \epsilon_{m p} d^{4}, \text { when } d \geq d_{0}
\end{array}\right.
$$

Where $d_{o}$ is the distance threshold for swapping amplification models, which can be calculated as $d_{o}=\sqrt{\frac{\epsilon_{f s}}{\epsilon_{m p}}}$

To receive an $l$ bits message the receiver expends:

$$
E_{R x}(l)=l E_{\text {elec }}
$$
as:

To aggregate $\mathrm{n}$ data signals of length $\mathrm{l}$ - bits, the energy consumption was calculated

$$
E_{D A-\operatorname{expend}}(l)=\ln E_{D A}
$$

\subsection{Network Model}

This section describes the network model and other basic assumptions:

1) $\mathrm{N}$ sensors are uniformly distributed within a square $3 \mathrm{D}$ rectangular field of area $A=$ $M \times M \times M$. The Base Station is positioned at the center of the square region. The number of sensor nodes $\mathrm{N}$ to be deployed depends specifically on the application.

2) All nodes are deployed randomly.

3) Each sensor can sense the environment in the 3D sphere of radius $r$.

4) All sensors are homogeneous, i.e., they have the same capacities.

5) All the sensor nodes have a particular identifier (ID) allocated to them. Each cluster head coordinates the MAC and routing of packets within their clusters. (see Figure 2)

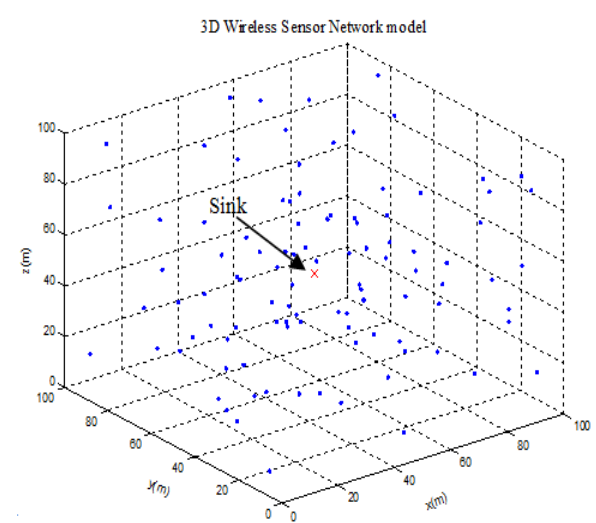

Figure 2. Three-dimensional Wireless Sensor Network model

\subsection{Optimal Number of Cluster}

We assume there are $N$ nodes distributed uniformly in $M \times M \times M \quad 3 \mathrm{D}$ region. If there are $c$ clusters, there are on average $N / c$ nodes per cluster. Each cluster-head dissipates energy receiving signals from the nodes and transmitting the aggregate signal to the base station. Therefore, the energy dissipated in the cluster-head node during a single frame is:

$$
E_{C H}=l \frac{N}{c} E_{\text {elec }}+l \frac{N}{c} E_{D A}+l \epsilon_{m p} d_{\text {toBS }}^{4}
$$

Where $l$ is the number of bits in each data message, $d_{t o B S}$ is the distance from the cluster head node to the BS, and we have assumed perfect data aggregation $E_{D A}$.

The expression for the energy spends by a non-cluster head is given by:

$$
E_{\text {nonCH }}=l E_{\text {elec }}+l \epsilon_{f s} d_{\text {toCH }}^{2}
$$

Where $d_{t o C H}$ is the distance from the node to the cluster head. 
Let $E\left[d_{t o B s}\right]$ be the Expected distance of cluster head from the base station. Assuming that the nodes are uniformly distributed, so it is calculated as follows:

$$
E\left[d_{\text {toBS }}^{2}\right]=\int_{0}^{x_{\max }} \int_{0}^{y_{\max }} \int_{0}^{z_{\max }}\left(x^{2}+y^{2}+z^{2}\right) f(x, y, z) d x d y d z
$$

Where $\mathrm{f}(\mathrm{x}, \mathrm{y}, \mathrm{z})$ is the probability density function of three dimensions random variable $X(x, y, z)$ which is uniform and given by:

$$
f=\frac{1}{V_{T}}=\frac{1}{M^{3}}
$$

If we assume that base station is the center of the network we can passing in the spherical coordinates:

$$
E\left[d_{t o B S}^{2}\right]=\int_{0}^{r_{\max }} \int_{0}^{\pi} \int_{0}^{2 \pi} r^{2} f(r, \theta, \varphi) r^{2} \sin \theta d r d \theta d \varphi
$$

The area of network is aspheric with radius $r_{\max }=M \times \sqrt[3]{3 / 4 \pi}$.

If the density of sensor nodes is uniform throughout the area then becomes independent of $\mathrm{r}, \theta$ and $\varphi$ then:

$$
E\left[d_{t O B S}^{2}\right]=\frac{3}{10}\left(\frac{3}{4 \pi}\right)^{\frac{2}{3}} M^{2}=0.5312 M^{2}
$$

The expected squared distance from the nodes to the cluster head (assumed to be at the center of mass of the cluster) is given by:

$$
E\left[d_{\text {toCH }}^{2}\right]=\int_{0}^{r_{\max }} \int_{0}^{\pi} \int_{0}^{2 \pi} r^{2} f(r, \theta, \varphi) r^{2} \sin \theta d r d \theta d \varphi
$$

If we assume this area is a sphere with radius $r_{\max }=M \times \sqrt[3]{3 / 4 \pi c}$ and $f(r, \theta, \varphi)$ is constant for $r, \theta$ and, (10) simplifies to:

$$
E\left[d_{t o C H}^{2}\right]=f \int_{0}^{M \times \sqrt[3]{3 / 4 \pi c}} \int_{0}^{\pi} \int_{0}^{2 \pi} r^{3} \sin \theta d r d \theta d \varphi
$$

If the density of nodes is uniform throughout the cluster area, then $f=c / M^{3}$ and:

$$
E\left[d_{\text {toCH }}^{2}\right]=\frac{3}{10} M^{2}\left(\frac{3}{4 \pi c}\right)^{\frac{2}{3}}
$$

Therefore, the total energy dissipated in the network per round, $E_{\text {Total }}$, is expressed by:

$$
E_{\text {Total }}=c E_{\text {cluster }}
$$

Where $E_{\text {cluster }}$ is the energy dissipated in cluster which giving by:

$$
E_{\text {Cluster }}=E_{C H}+\left(\frac{N}{c}-1\right) E_{\text {nonCH }} \approx E_{C H}+\frac{N}{c} E_{\text {nonCH }}
$$

This can be calculated by:

$$
E_{\text {Cluster }}=l\left(\frac{N}{c} E_{\text {elec }}+\frac{N}{c} E_{D A}+\epsilon_{m p} d_{\text {toBS }}^{4}\right)+l\left(\frac{N}{c} E_{\text {elec }}+\frac{N}{c} \epsilon_{f s} d_{\text {toCH }}^{2}\right)
$$

Therefore, the total energy dissipated in the network is simplified by:

$$
E_{\text {Total }}=l\left(2 N E_{\text {elec }}+N E_{D A}+c \epsilon_{m p} d_{\text {toBS }}^{4}+N \epsilon_{f s} \frac{3}{10} M^{2}\left(\frac{3}{4 \pi c}\right)^{\frac{2}{3}}\right)
$$


We can find the optimum number of clusters by setting the derivative of $E_{\text {Total }}$ with respect to $\mathrm{c}$ to zero.

$$
\begin{aligned}
& \frac{\partial E_{T o t a l}}{\partial c}=0 \\
& C_{o p t}=0.2147 \times\left(N \frac{\epsilon_{f s}}{\epsilon_{m p}} \frac{M^{2}}{d_{\text {toBS }}^{4}}\right)^{\frac{3}{5}}
\end{aligned}
$$

The optimal probability for becoming a cluster-head can also be computed as:

$$
P_{o p t}=\frac{C_{o p t}}{N}
$$

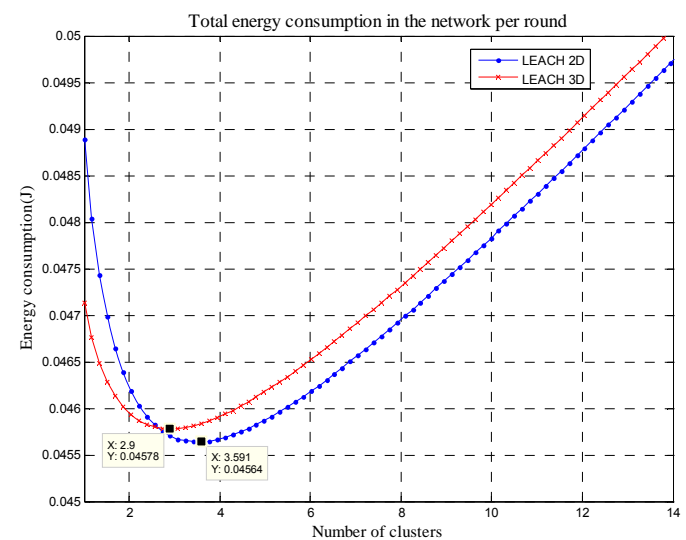

Figure 3. Variation of energy consumption for different values of clusters number $c$

In Figure 3, we show the average energy consumption per round by each sensor node as a function of the number of clusters for two types of model, 3D and 2D WSN. Firstly, graph of the LEACH 3D model follow the same of LEACH 2D. Secondly, the graph of LEACH 3D model shows that the simulation agrees well with the analysis results. In the other hand, the 3D model consumes more energy than the $2 \mathrm{D}$ model which depends essentially to the no negligible value of the network height. However, this model has an optimal number of clusters less than the other model which can exploit advantageous to minimize the lifetime of the network.In this section, it is explained the results of research and at the same time is given the comprehensive discussion. Results can be presented in figures, graphs, tables and others that make the reader understand easily $[2,5]$. The discussion can be made in several sub-chapters.

\section{Simulation Results}

\subsection{Parameter Settings}

In this section, we study the performance of LEACH 3D protocol under different scenarios using MATLAB. We consider a model illustrate in the Figure 2 with $\mathrm{N}=100$ nodes randomly and uniformly distributed in a $100 \mathrm{~m} \times 100 \mathrm{~m} \times 100 \mathrm{~m}$ field. To compare the performance of LEACH 3D with LEACH 2D protocol, we ignore the effect caused by signal collision and interference in the wireless channel. The radio parameters used in our simulations are shown in Table 1. 
Table 1. Energy Model Parameters

\begin{tabular}{|c|c|}
\hline Parameter & Value \\
\hline Initial Node Energy & $0.5 \mathrm{~J}$ \\
\hline $\mathrm{N}$ & 100 \\
\hline$P$ & 0.05 \\
\hline $\mathrm{E}_{\text {elec }}$ & $50 \mathrm{~nJ} / \mathrm{bit}$ \\
\hline $\mathrm{E}_{\mathrm{DA}}$ & $5 \mathrm{pJ} / \mathrm{bit}$ \\
\hline$\epsilon_{\mathrm{fs}}$ & $10 \mathrm{pJ} / \mathrm{bit} / \mathrm{m}^{2}$ \\
\hline$\epsilon_{\mathrm{mp}}$ & $0.0013 \mathrm{pJ} / \mathrm{bit} / \mathrm{m}^{4}$ \\
\hline $\mathrm{d}_{\mathrm{toBS}}$ & $100 \mathrm{~m}$ \\
\hline$l$ & 500 Bytes \\
\hline Rounds & 2000 \\
\hline
\end{tabular}

\subsubsection{Simulation metrics}

Performance metrics used in the simulation study are:

1) Energy consumption analysis

2) Number of alive nodes per round.

3) Percentage of Node death

4) Throughput

5) Decrease:

$$
\text { Increase }=\frac{F N D \text { of } L E A C H 3 D-F N D \text { of } L E A C H 2 D}{F N D \text { of } L E A C H 2 D} \times 100
$$

\subsection{Simulation Results}

\subsubsection{Energy consumption analysis}

The performance of LEACH 3D is compared with that of the original LEACH in terms of energy and is shown in Figure 4. With the use of 3D deployment of nodes, the energy consumption of the network is decreased. This is due to the gain of the energy dissipated by height of network. From the graph it is clear that LEACH 3D decrease twice the energy savings than LEACH protocol.

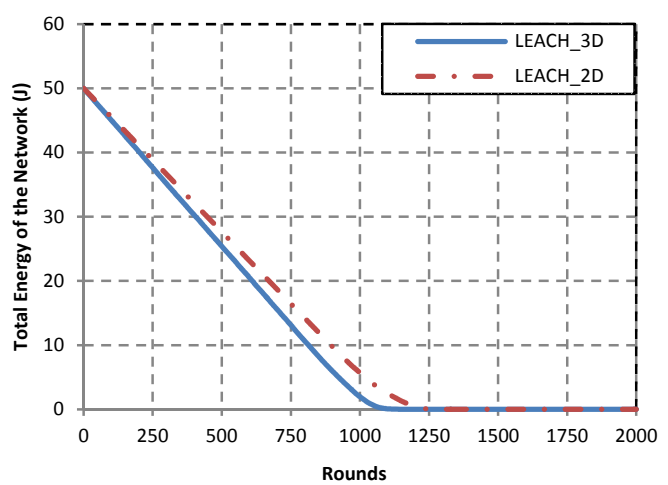

Figure 4. Energy analysis comparison of LEACH 3D and LEACH 2D

\subsubsection{Network Lifetime}

The number of nodes alive for each round of data transmission is observed for the LEACH 2D and 3D protocols to evaluate the lifetime of the network. Figure 5 and Figure 6 show the performance of LEACH 3D compared to LEACH 2D. It is observed that the LEACH 3D is less perform than LEACH 2D due to energy dissipation of individual node throughout the network which depend essentially on the distance between nodes and sink. 


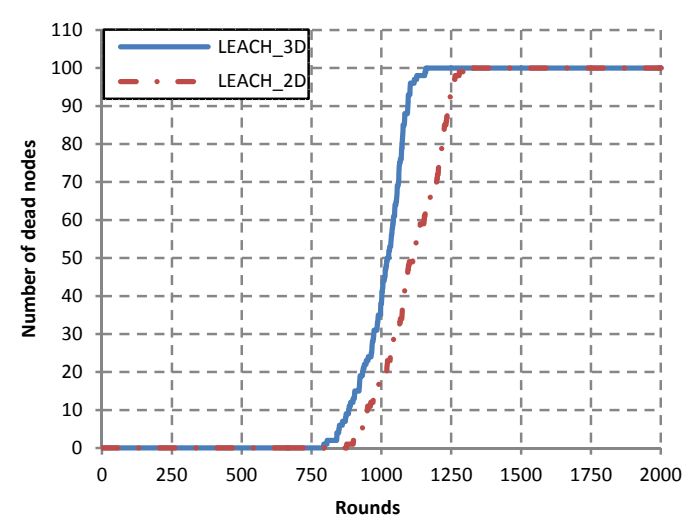

Figure 5. Number of dead nodes per round comparison of LEACH 3D and LEACH 2D

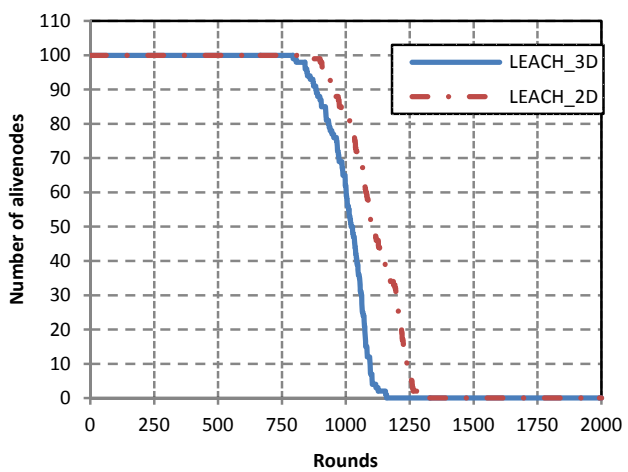

Figure 6. Number of alive nodes per round comparison of LEACH 3D and LEACH 2D

\subsubsection{Throughput}

Referred to Figure 7 , it show clearly that LEACH 3D provide a poor throughput compared to LEACH 2D protocol, this decrease is justified by the low lifetime which give the three dimensional deployment of the nodes in the network.

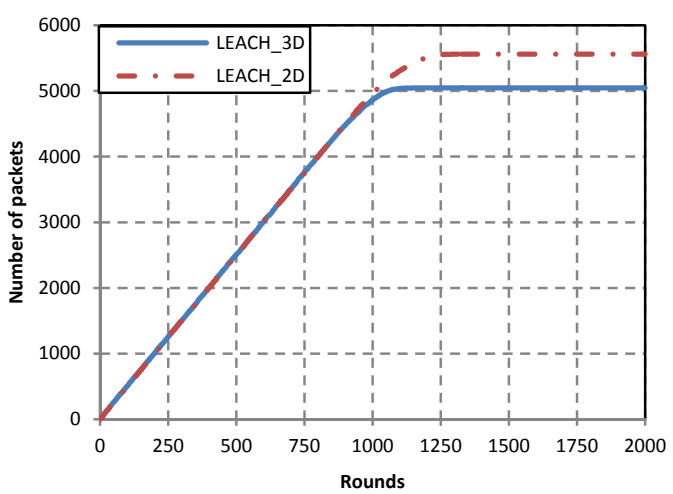

Figure 7. Performance of the protocols

\subsubsection{Decrease}

Generally, we can illustrate the decrease of the LEACH 3D in the Figure 8. It's noted that the throughput decreases $21 \%$ as much than LEACH $2 \mathrm{D}$ due to its less energy. Whereas, LEACH 2D outperforms the FND of LEACH 2D by $21 \%$ and by $28 \%$ for LND. In the other hand, LEACH 3D consumes $32 \%$ more energy than LEACH $2 \mathrm{D}$. 
LEACH 3D and LEACH 2D increase

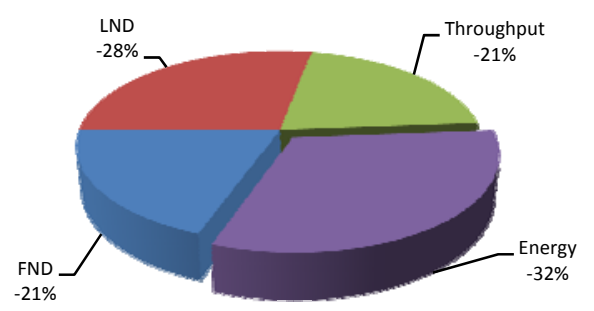

Figure 8. Decrease of LEACH 3D compared to LEACH 2D.

\subsection{Result Analysis}

From our simulations, we observed that LEACH 3D consumes more energy and delivers less packets to the base station. These results can be interpreted by the difference of distance between nodes in both situations which naturally causes by the random deployment of nodes.

\section{Conclusion and Future Work}

Recently, 3D wireless sensor networks have known a great prevalent due to their large applications such as underwater, space communications, atmospheric, forest or building. The analytic of 3D WSN is more complexity than the analytic in 2D WSN. Therefore, many researches project the 3D WSN in $2 \mathrm{D}$ WSN. In this paper, we demonstrate by simulation, that this approximation is not reasonable if the height of network is greater than length and breadth of this network. We strongly persuaded that projection of WSN in 2D environment is unjustifiable in reason that the 3D WSN is much closer to our physical word. As future work, we will work to optimize the energy consumption of this network, since the number of cluster head in 3D WSN gives more result than 2D WSN.

\section{References}

[1] HM Ammari, SK Das. Coverage and connectivity in three-dimensional wireless sensor networks using percolation theory. IEEE Trans. Parallel Distrib. Syst. (IEEE TPDS). 2009; 20(6).

[2] Kay Romer, Friedemann Mattern. The Design Space of Wireless Sensor Networks. IEEE Wireless Communications. 2004; 11(6): 54-61.

[3] Wendi R Heinzelman, Anantha Chandrakasan, Hari Balakrishnan. Energy efficient communication protocol for wireless microsensor networks. IEEE International Conference on System Sciences. 2000: 1-10.

[4] W Heinzelman, A Chandrakasan, $\mathrm{H}$ Balakrishnan. An application specific protocol architecture for wireless microsensor networks. IEEE Transactions on Wireless Communications. 2002; 1(4): 660670.

[5] Xia Li, Yongqian Wang, Jingjin Zhou. An energy-efficient clustering algorithm for underwater acoustic sensor networks. Control Engineering and Communication Technology (ICCECT), 2012 International Conference. 2012: 711-714.

[6] X Li, SL Fang, YC Zhang. The study on clustering algorithm of the underwater acoustic sensor networks. The 14th International Conference on Mechatronics and Machine Vision in Practice (M2VIP 2007). 2007.

[7] Guangsong Yang, Mingbo Xiao, En Cheng, Jing Zhang. A cluster-head selection scheme for underwater acoustic sensor networks. Communications and Mobile Computing (CMC), 2010 International Conference. 2010; 3: 188-191.

[8] Liu G, Wei C. A new multi-path routing protocol based on cluster for underwater acoustic sensor networks. International Conference on Multimedia Technology (ICMT). 2011: 91-94.

[9] P Wang, C Li, J Zheng. Distributed minimum-cost clustering protocol for underwater sensor networks (UWSNs). IEEE International Conference on Communications (ICC 2007). 2007: 3510-3515.

[10] Salva-Garau F, Stojanovic M. Multi-cluster protocol for ad hoc mobile underwater acoustic networks. OCEANS 2003 Proceedings. 2003; 1(26): 91-98. 
[11] X Gu, Y Jin, Y Sun, J Yan. Maximum lifetime routing strategies for wireless sensor networks in coal mine. In 2010 International Conference on Computer Engineering and Technology. 2010: 341-344.

[12] ZC Zhu, GB Zhou, GZ Chen. Chain-type wireless underground mine sensor networks for gas monitoring. Advanced Science Letters. 2011; 4(2): 391-399.

[13] GB Zhou, ZC Zhu, GZ Chen, NN Hu. Energy-efficient chain-type wireless sensor network for gas monitoring. In International Conference on Information and Computing Science. 2009: 125-128. 